\title{
Inside-Out Bulge Formation and the Origin of the Hubble Sequence
}

\author{
By Frank C. van den Bosch \\ Department of Astronomy, University of Washington, Box 351580, Seattle, WA 98195, USA
}

\begin{abstract}
Galactic disks are thought to originate from the cooling of baryonic material inside virialized dark halos. In order for these disks to have scalelengths comparable to observed galaxies, the specific angular momentum of the baryons has to be largely conserved. Because of the spread in angular momenta of dark halos, a significant fraction of disks are expected to be too small for them to be stable, even if no angular momentum is lost. Here it is suggested that a selfregulating mechanism is at work, transforming part of the baryonic material into a bulge, such that the remainder of the baryons can settle in a stable disk component. This inside-out bulge formation scenario is coupled to the Fall \& Efstathiou theory of disk formation to search for the parameters and physical processes that determine the disk-to-bulge ratio, and therefore explain to a large extent the origin of the Hubble sequence. The Tully-Fisher relation is used to normalize the fraction of baryons that forms the galaxy, and two different scenarios are investigated for how this baryonic material is accumulated in the center of the dark halo. This simple galaxy formation scenario can account for both spirals and S0s, but fails to incorporate more bulge dominated systems.
\end{abstract}

\section{Introduction}

Despite considerable progress in our understanding of the formation of galaxies, the origin of the Hubble sequence remains a major unsolved problem. The main morphological parameter that sets the classification of galaxies in the Hubble diagram is the disk-tobulge ratio $(D / B)$. Understanding the origin of the Hubble sequence is thus intimately related to understanding the parameters and processes that determine the ratio between the masses of disk and bulge. Especially, we need to understand whether this ratio is imprinted in the initial conditions ('nature') or whether it results from environmental processes such as mergers and impulsive collisions ('nurture').

Here I suggest a simple inside-out formation scenario for the bulge (a 'nature'-variant) and investigate the differences in properties of the proto-galaxies that result in different disk-to-bulge ratios. A more detailed discussion on the background and ingredients of the models can be found in van den Bosch (1998; hereafter vdB98).

\section{The formation scenario}

In the standard picture of galaxy formation, galaxies form through the hierarchical clustering of dark matter and subsequent cooling of the baryonic matter in the dark halo cores. Coupled with the notion of angular momentum gain by tidal torques induced by nearby proto-galaxies, this theory provides the background for a model for the formation of galactic disks. In this model, the angular momentum of the baryons is assumed to be conserved causing the baryons to settle in a rapidly rotating disk (e.g., Fall \& Efstathiou 1980). The turn-around, virialization, and subsequent cooling of the baryonic matter of a proto-galaxy is an inside-out process. First the innermost shells virialize and heat its baryonic material to the virial temperature. The cooling time of this dense, inner material is very short, whereas its specific angular momentum is relatively low. If the cooling time of the gas is shorter than the dynamical time, the gas will condense in 
clumps that form stars, and this clumpiness is likely to result in a bulge. Even if the low-angular momentum material accumulates in a disk, the self-gravity of such a small, compact disk makes it violently unstable, and transforms it into a bar. Bars are efficient in transporting gas inwards, and can cause vertical heating by means of a collective bending instability. Both these processes lead ultimately to the dissolution of the bar; first the bar takes a hotter, triaxial shape, but is later transformed in a spheroidal bulge component. There is thus a natural tendency for the inner, low angular momentum baryonic material to form a bulge component rather than a disk. Because of the ongoing virialization, subsequent shells of material cool and try to settle into a disk structure at a radius determined by their angular momentum. If the resulting disk is unstable, part of the material is transformed into bulge material. This process of disk-bulge formation is self-regulating in that the bulge grows until it is massive enough to sustain the remaining gas in the form of a stable disk. I explore this inside-out bulge formation scenario, by incorporating it into the standard Fall \& Efstathiou theory for disk formation.

The ansatz for the models are the properties of dark halos, which are assumed to follow the universal density profiles proposed by Navarro, Frenk \& White (1997), and whose halo spin parameters, $\lambda$, follow a log-normal distribution in concordance with both numerical and analytical studies. I assume that only a certain fraction, $\epsilon_{\mathrm{gf}}$, of the available baryons in a given halo ultimately settles in the disk-bulge system. Two extreme scenarios for this galaxy formation (in)efficiency are considered. In the first scenario, which I call the 'cooling'-scenario, only the inner fraction $\epsilon_{\mathrm{gf}}$ of the baryonic mass is able to cool and form the disk-bulge system: the outer parts of the halo, where the density is lowest, but which contain the largest fraction of the total angular momentum, never gets to cool. In the second scenario, referred to hereafter as the 'feedback'-scenario, the processes related to feedback and star formation are assumed to yield equal probabilities, $\epsilon_{\mathrm{gf}}$, for each baryon in the dark halo, independent of its initial radius or specific angular momentum, to ultimately end up in the disk-bulge system. The values of $\epsilon_{\mathrm{gf}}$ are normalized by fitting the model disks to the zero-point of the observed Tully-Fisher relation. Recent observations of high redshift spirals suggest that the zero-point of the Tully-Fisher relation does not evolve with redshift. This implies that the galaxy formation efficiency, $\epsilon_{\mathrm{gf}}$, was higher at higher redshifts (see vdb98 for details). Disks are modeled as exponentials with a scalelength proportional to $\lambda$ times the virial radius of the halo (as in the disk-formation scenario of Fall \& Efstathiou). The bulge mass is determined by requiring that the disk is stable. Since the amount of self-gravity of the disk is directly related to the amount of angular momentum of the gas, the disk-to-bulge ratio in this scenario is mainly determined by the spin parameter of the dark halo out of which the galaxy forms.

\section{Clues to the formation of bulge-disk systems}

Constraints on the formation scenario envisioned above can be obtained from a comparison of these disk-bulge-halo models with real galaxies. From the literature I compiled a list of $\sim 200$ disk-bulge systems, including a wide variety of galaxies: both high and low surface brightness spirals (HSB and LSB respectively), S0, and disky ellipticals (see vdB98 for details). After choosing a cosmology and a formation redshift, $z$, I calculate, for each galaxy in this sample, the spin parameter $\lambda$ of the dark halo which, for the assumptions underlying the formation scenario proposed here, yields the observed disk properties (scale-length and central surface brightness). We thus use the formation scenario to link the disk properties to those of the dark halo, and use the known statistical properties of dark halos to discriminate between different cosmogonies.

The main results are shown in Figure 1, where I plot the inferred values of $\lambda$ versus 


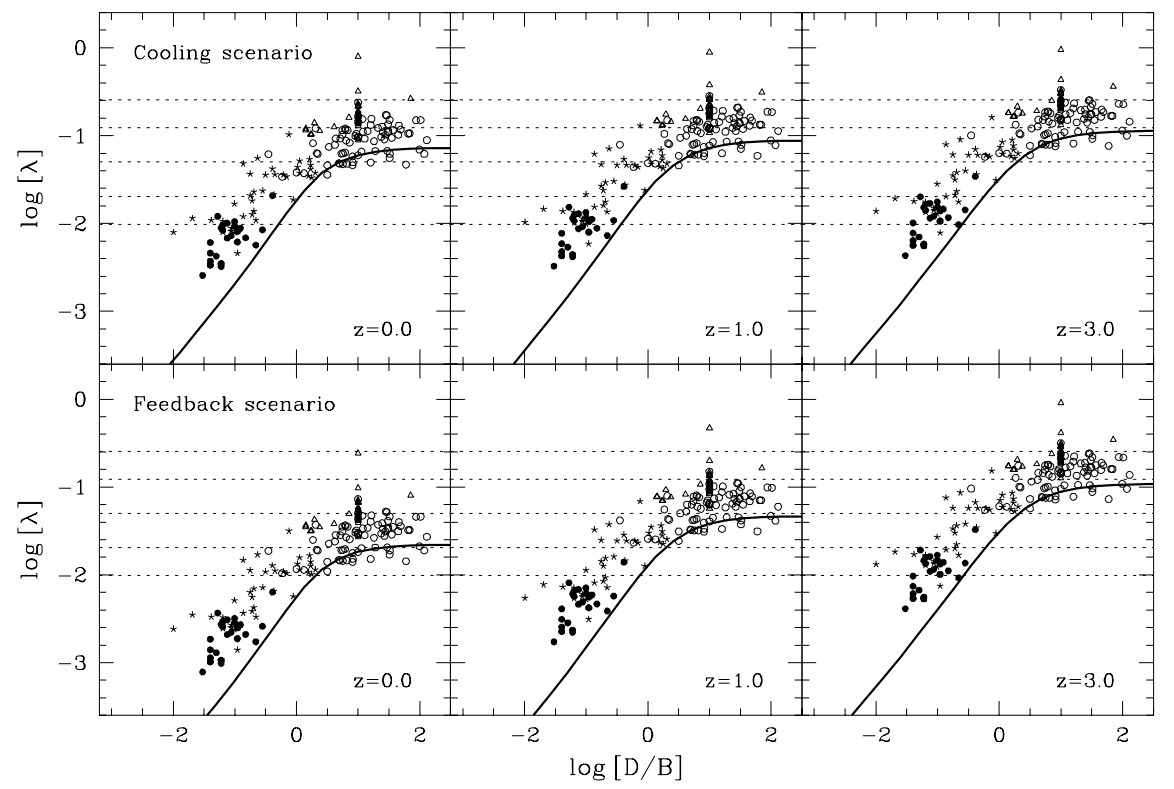

Figure 1. Results for a OCDM cosmology with $\Omega_{0}=0.3$. Plotted are the logarithm of the spin parameter versus the logarithm of the disk-to-bulge ratio. Solid circles correspond to disky ellipticals, stars to S0s, open circles to HSB spirals, and triangles to LSB spirals. The thick solid line is the stability margin; halos below this line result in unstable disks. As can be seen, real disks avoid this region, but stay relatively close to the stability margin, in agreement with the self-regulating bulge formation scenario proposed here. The dashed curves correspond to the 1, 10, 50, 90, and 99 percent levels of the cumulative distribution of the spin parameter. Upper panels correspond to the cooling scenario, and lower panels to the feedback scenario. Panels on the left correspond to $z=0$, middle panels to $z=1$, and panels on the right to $z=3$.

the observed disk-to-bulge ratio for the galaxies in the sample. The dotted lines outline the distribution function of halo spin parameters of dark halos; it can thus be inferred what the predicted distribution of disk-to-bulge ratios is for galaxies that form at a given formation redshift. Results are presented for an open cold dark matter (OCDM) model with $\Omega_{0}=0.3$ and no cosmological constant $\left(\Omega_{\Lambda}=0\right)$. These results are virtually independent of the value of $\Omega_{\Lambda}$, but depend strongly on $\Omega_{0}$, which sets the baryon mass fraction of the Universe. Throughout, a universal baryon density of $\Omega_{b}=0.0125 h^{-2}$ is assumed, in agreement with nucleosynthesis constraints. The inferred spin parameters are larger for higher values of the assumed formation redshifts. This owes to the fact that halos that virialize at higher redshifts are denser. Since the scalelength of the disk is proportional to $\lambda$ times the virial radius of the halo, higher formation redshifts imply larger spin parameters in order to yield the observed disk scalelength. In the cooling scenario, the probability that a certain halo yields a system with a large disk-to-bulge ratio (e.g., a spiral) is rather small. This is due to the fact that in this scenario most of the high angular momentum material never gets to cool to become part of the disk. The large observed fraction of spirals in the field, renders this scenario improbable. For the feedback cosmogony, however, a more promising scenario unfolds: At high redshifts $(z \gtrsim 1)$ the majority of halos yields systems with relatively small disks (e.g., S0s), whereas systems that form more recently are more disk-dominated (e.g., spirals). This difference owes to two effects. First of all, halos at higher redshifts are denser, and secondly, the redshift independence of the Tully-Fisher relation implies that $\epsilon_{\mathrm{gf}}$ was higher at higher redshifts. Coupled to the notion that proto-galaxies that collapse at high redshifts are 
preferentially found in overdense regions such as clusters, this scenario thus automatically yields a morphology-density relation, in which S0s are predominantly formed in clusters of galaxies, whereas spirals are more confined to the field.

\section{Conclusions}

- Inside-out bulge formation is a natural by-product of the Fall \& Efstathiou theory for disk formation.

- Disk-bulge systems do not have bulges that are significantly more massive than required by stability of the disk component. This suggests a coupling between the formation of disk and bulge, and is consistent with the self-regulating, inside-out bulge formation scenario proposed here.

- A comparison of the angular momenta of dark halos and spirals suggests that the baryonic material that builds the disk can not loose a significant fraction of its angular momentum. This rules against the 'cooling scenario' envisioned here, in which most of the angular momentum remains in the baryonic material in the outer parts of the halo that never gets to cool.

- If we live in a low-density Universe $\left(\Omega_{0} \lesssim 0.3\right)$, the only efficient way to make spiral galaxies is by assuring that only a relatively small fraction of the available baryons make it into the galaxy, and furthermore that the probability that a certain baryon becomes a constituent of the final galaxy has to be independent of its specific angular momentum, as described by the 'feedback scenario'.

- If more extended observations confirm that the zero-point of the Tully-Fisher relation is independent of redshift, it implies that the galaxy formation efficiency, $\epsilon_{\mathrm{gf}}$, was higher at earlier times. Coupled with the notion that density perturbations that collapse early are preferentially found in high density environments such as clusters, the scenario presented here then automatically predicts a morphology-density relation in which S0s are most likely to be found in clusters.

- A reasonable variation in formation redshift and halo angular momentum can yield approximately one order of magnitude variation in disk-to-bulge ratio, and the simple formation scenario proposed here can account for both spirals and S0s. However, disky ellipticals have too large bulges and too small disks to be incorporated in this scenario. Apparently, their formation and/or evolution has seen some processes that caused the baryons to loose a significant amount of their angular momentum. Merging and impulsive collisions (e.g., galaxy harassment) are likely to play a major role for these systems.

It thus seems that both 'nature' and 'nurture' are accountable for the formation of spheroids, and that the Hubble sequence has a hybrid origin.

Support for this work was provided by NASA through Hubble Fellowship grant \# HF-01102.11-97.A awarded by the Space Telescope Science Institute, which is operated by AURA for NASA under contract NAS 5-26555.

\section{REFERENCES}

FAll, S. M., \& Efstathiou, G. 1980 Formation and rotation of disc galaxies with haloes MNRAS 193, 189-206

Navarro, J. F., Frenk, C. S., \& White, S. D. M. 1997 A universal density profile from hierarchical clustering ApJ 490, 493-508

VAN DEN Bosch, F. C. 1998 The formation of disk-bulge-halo systems and the origin of the Hubble sequence ApJ 507, 601-614 